\title{
Anatomie descriptive et fonctionnelle de l'articulation temporo-mandibulaire
}

\author{
Descriptive and functional anatomy \\ of the temporomandibular joint
}

\section{Résumé}

\section{MOTS-CLEFS :}

- ATM, ptérygoïdien latéral, disque, muscles masticateurs, cinétique mandibulaire, occlusion, nerf mandibulaire, processus condylaire, dysfonctions de l'ATM, syndrome Algodysfonctionnel de l'Appareil Manducateur, bruxisme

\section{KEYWORDS:}

- TMJ, lateral pterygoid, disc, mastication muscles, mandibular kinetics, occlusion, mandibular nerve, condyloid process, TMJ dysfunctions, myofascial poin dysfunction syndrome, bruxism.

AOS 2013;265:4-18 DOI: $10.1051 / \mathrm{aos} / 2013502$ (C) EDP Sciences 2013
L'articulation temporo-mandibulaire (ATM) est une articulation complexe, les surfaces articulaires non concordantes s'adaptant grâce au tendon du muscle ptérygoïdien latéral qui coulisse entre elles lors de tous les mouvements mandibulaires. Il n'existe pas de parallélisme anatomoclinique entre l'importance de l'atteinte articulaire et la gravité des symptômes ressentis par le patient. L'anatomie descriptive de l'ATM est bien connue, malgré une certaine variabilité, mais son aspect dynamique l'est moins en l'absence d'un système d'imagerie adapté au suivi tridimensionnel d'un sujet en temps réel. L'abord pluridisciplinaire de la pathologie des dysfonctions de l'ATM a fait progresser la connaissance de l'anatomie fonctionnelle de cette articulation. Le nerf mandibulaire en assure l'innervation motrice et sensitive pouvant initier un cercle vicieux entre spasmes et douleurs. L'imputabilité des symptômes otologiques, présents lors de certains cas de dysfonctions de l'ATM, ne peut être retenue de façon systématique.
Abstract

The temporomandibular joint (TMJ) is a complex joint, with the mismatched articular surfaces adapting to one another via the tendon of the lateral pterygoid muscle which slides between them during all jaw movements. There are no anatomical or clinical correlations between the significance of a joint condition and the severity of symptoms experienced by the patient. The descriptive anatomy of the TMJ is well-known despite some variability, but its dynamic aspects are less so due to the lack of an imaging system adapted to three-dimensional follow-up of an individual in real time. A multidisciplinary approach to TMJ dysfunctions has prompted a more in-depth knowledge on the functional anatomy of this joint to be developed. Motor and sensory innervation is derived from the mandibular nerve, which may lead to a vicious circle between spasms and pain. A causal relationship with otological symptoms present in some cases of TMJ dysfunctions cannot be considered as standard.

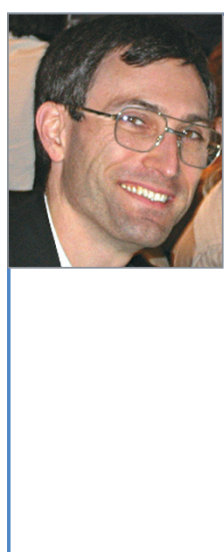

\section{- Christophe Bonnefoy}

Chargé d'enseignement à l'université Paris VI,

service de stomatologie et de chirurgie maxillo-faciale UFR 056 Pitié-Salpêtrière, lauréat de l'Académie nationale de chirurgie-dentaire.

\section{- Luc Chikhani,}

Praticien hospitalier, chirurgien des hôpitaux, responsable de l'unité de stomatologie et chirurgie maxillofaciale de l'hôpital européen Georges Pompidou, chargé d'enseignement à l'université UFR Odontologie, Paris VII.

\section{- Jacques Dichamp,}

Praticien hospitalier stomatologiste chargé d'enseignement

à l'université Paris VI, service de stomatologie et de chirurgie maxillofaciale,

UFR 056 Pitié-Salpêtrière et à l'hôpital européen Georges Pompidou. 


\section{INTRODUCTION}

Une bonne connaissance de l'anatomie de l'articulation temporo-mandibulaire (ATM) est indispensable au thérapeute s'il ne désire pas se contenter dêtre un prescripteur d'antalgiques et de myorelaxants [2]. Lanatomie de cette articulation est donc non seulement primordiale pour l'anatomiste mais également pour le clinicien puisque, sans elle, il est illusoire d'en comprendre le fonctionnement. Cet article tente de faire le point sur les données acquises de la science anatomique afin de les relier à la clinique et aux dysfonctions de lappareil manducateur.

\section{RAPPELS ANATOMIQUES}

\section{Généralités}

L'ATM relie la mandibule au massif facial et réalise l'union d'une partie convexe, le processus condylaire mandibulaire mobile, et d'une partie concave fixe, la fosse mandibulaire de l'os temporal se poursuivant en avant par une partie convexe, le tubercule articulaire du temporal, par l'intermédiaire d'un disque biconcave. Sans lexistence de ce disque, cette articulation serait fonctionnellement instable. L'ATM est une articulation en suspension et par définition non soumise à des appuis par la force gravitationnelle.

Les deux articulations droite et gauche sont seules dans l'organisme à travailler de façon couplée, « en stéréo ", la mandibule les reliant entre elles à la manière d'un guidon. Elles font partie intégrante de l'appareil manducateur, système complexe composé déléments passifs ostéo-ligamento-articulaires et dentaires et déléments actifs fonctionnels musculaires.

L'ATM est issue du mésenchyme du premier arc branchial lié au territoire d'innervation du nerf trijumeau. Le processus cartilagineux mandibulaire devient le cartilage de Meckel, entièrement formé à la fin de la période embryonnaire, qui sert de guide à la croissance mandibulaire et forme deux osselets de loreille moyenne : le malleus et l'incus.

L'ATM est une articulation fonctionnelle avant édification totale in utero puisqu'on observe une succion du pouce à 4 mois et demi en collaboration avec le peaucier antérieur facial [2].

\section{OSTÉOLOGIE}

\section{Os temporal (Fig. 1)}

\section{Généralités}

La surface articulaire de l'os temporal est constituée de la fosse mandibulaire et du tubercule articulaire.

\section{Fosse mandibulaire}

La portion horizontale de lécaille du temporal est creusée par une forte dépression elliptique, concave vers le bas, à grand axe oblique en avant et en dedans : la fosse mandibulaire. Elle est limitée en arrière par le méat acoustique externe et le tubercule zygomatique postérieur, en avant par le tubercule articulaire,

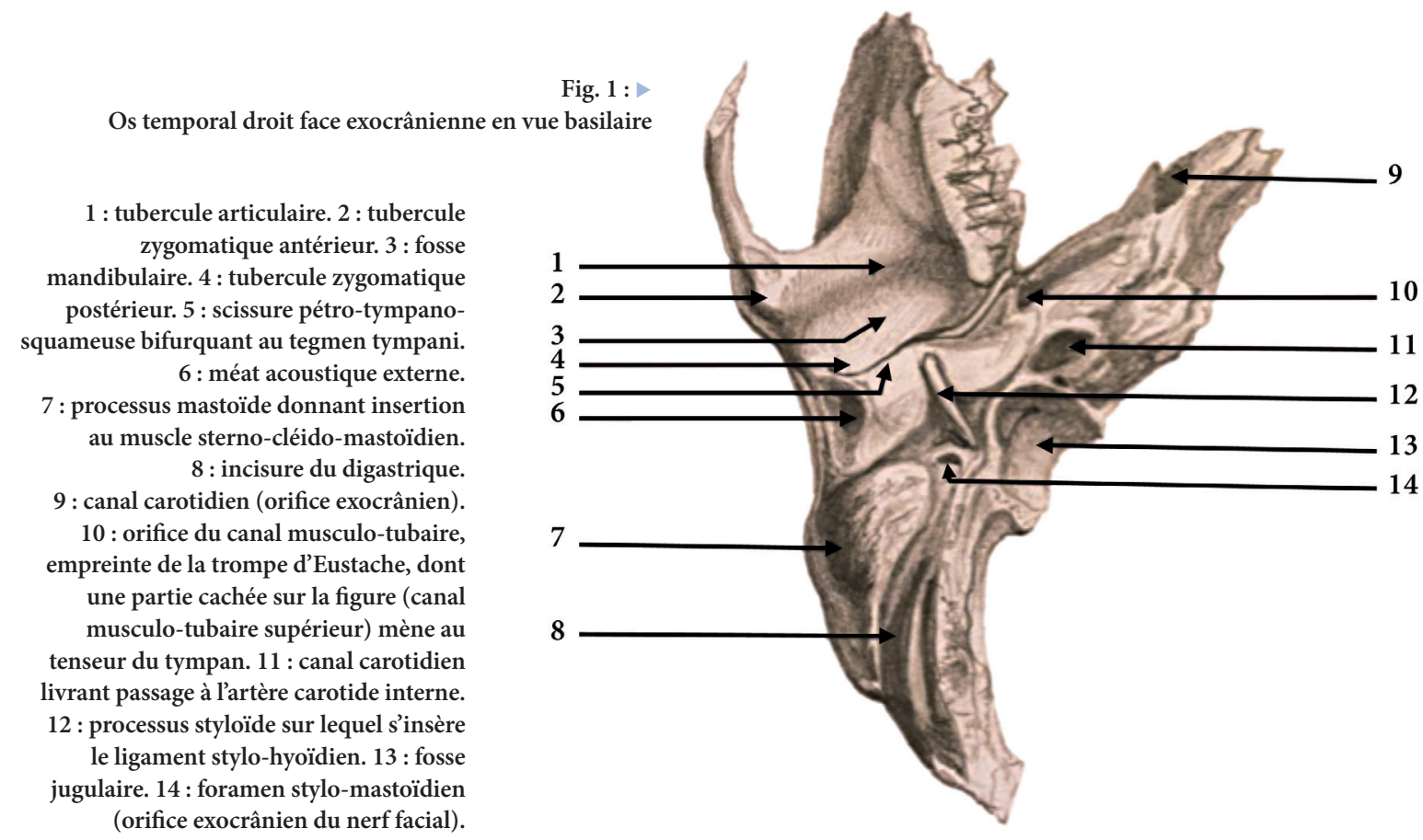




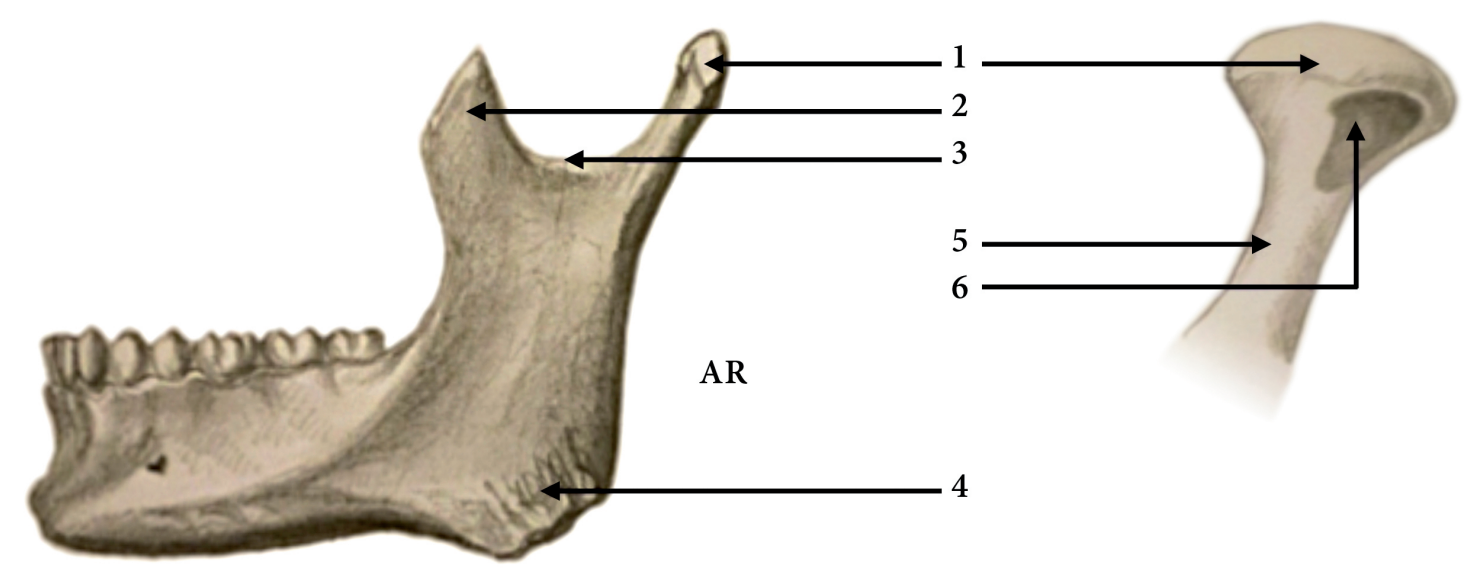

$\triangle$ Fig. 2 :

BAS

Os mandibulaire face latérale et détail du processus condylaire en vue antéro-médiale afin de bien mettre en évidence l'insertion du ptérygoïdien latéral.

1 : processus condylaire. 2 : processus coronoïde. 3 : incisure mandibulaire. 4 : crêtes d'insertion du muscle masséter et angle goniaque en partie basse du ramus ou branche montante (hyperostose angulo-mandibulaire en cas d'hypertrophie du masséter chez le bruxomane). 5 : col du processus condylaire. 6 : fossette ptérygö̈dienne.

en dehors par la racine longitudinale du processus zygomatique et en dedans par l'épine du sphénoïde. Elle est divisée en deux parties par le prolongement latéral tympano-squameux (scissure de Glaser) de la scissure pétro-tympano-squameuse qui divise la fosse mandibulaire en deux champs, l'un antérieur squameux recouvert de tissu articulaire fibreux et l'autre postérieur tympanal non articulaire.

Sur la scissure pétro-tympano-squameuse s'insère en arrière l'aponévrose interptérygoïdienne et en avant la capsule de l'ATM. Cette scissure, normalement fermée, conduit au tympan et au processus gracilis du malleus.

\section{Tubercule articulaire}

Il est schématiquement semblable à une sorte de cylindre grossier à grand axe à peu près parallèle à celui de la fosse mandibulaire, c'est-à-dire oblique en avant et en dedans. Il se termine en dehors au niveau du tubercule zygomatique antérieur à la jonction avec la racine longitudinale du zygoma. Il est convexe d'avant en arrière et transversalement parce qu'il est recouvert de tissu articulaire fibreux.

\section{Os mandibulaire (Fig. 2)}

\section{Généralités}

Le processus condylaire s'articule avec le temporal. Sa morphologie subit un remodelage fonctionnel (déglutition, mastication, habitudes fonctionnelles) allant du globuleux vers l'adaptatif [2].

\section{Processus condylaire}

Le processus condylaire est une éminence ovoïde, d'environ $2 \mathrm{~cm}$ par $1 \mathrm{~cm}$, qui fait pendant à la fosse mandibulaire et s'aligne selon un grand axe transversal oblique en arrière et en dedans. Les deux axes des processus condylaires droit et gauche se croisent au niveau du tiers antérieur du foramen magnum selon un angle qui varie de 130 à $140^{\circ}$.

Il présente un pôle latéral et un pôle médial, avec deux versants séparés par une crête mousse parallèle au grand axe du condyle. Le versant antérieur, le plus important en surface, est recouvert de cartilage et constitue une surface articulaire convexe vers le haut et allongée transversalement. Il est fonctionnel puisqu'en rapport avec le disque de l'articulation. Le versant postérieur est verticalisé, dépourvu de cartilage et non fonctionnel.

Le processus condylaire est déjeté en médial dans le sens transversal et surplombe le col condylaire grêle et la face médiale de la branche montante sousjacente qui le prolonge. Sous ce surplomb, en position antéro-médiale, se trouve la fossette ptérygoïdienne qui donne insertion au faisceau inférieur du muscle ptérygoïdien latéral.

\section{MYOLOGIE}

Les muscles en rapports étroits avec l'ATM, soit 4 muscles pairs et symétriques, sont issus, phylogénétiquement, d'une même masse musculaire (les autres 


\begin{tabular}{|c|c|c|}
\hline MUSCLE & SITUATION & DESCRIPTION \\
\hline Masséter (M) & $\begin{array}{l}\text { Muscle court, épais, quadrilatère, sous- } \\
\text { jacent au muscle Temporal, appliqué par trois } \\
\text { faisceaux, profond, moyen et superficiel, tendus } \\
\text { de l'arcade zygomatique à la face latérale du } \\
\text { ramus et du processus coronoïde }(15,8) \text {. }\end{array}$ & $\begin{array}{l}\text { Faisceau superficiel : oblique en bas et en arrière. Faisceau moyen } \\
\text { vertical. } \\
\text { Faisceau profond du } M \text { prolongeant le temporal sur la face latérale } \\
\text { du processus coronoïde et envoyant une expansion postérieure sur la } \\
\text { lame tendineuse pré-discale. }\end{array}$ \\
\hline Temporal (T) & $\begin{array}{l}\text { Il forme un éventail de profil au niveau de la } \\
\text { fosse temporale dont les fibres convergent vers } \\
\text { le processus coronoïde de la mandibule }(15,10) \text {. }\end{array}$ & $\begin{array}{l}\text { Le tendon, né de la convergence de toutes ces fibres, passe dans le } \\
\text { foramen zygomatique et se termine sur le processus coronoïde et le } \\
\text { bord antérieur de la branche montante de la mabdibule jusqu'à la crête } \\
\text { buccinatrice. Il prolonge les fibres du masséter d'où l'appellation de } \\
\text { « complexe tempo-massétérique ». Une expansion des fibres pos- } \\
\text { térieures s'insère sur la lame tendineuse prédiscale médialement au } \\
\text { masséter et latéralement au faisceau supérieur du Ptérygoïdien Latéral. }\end{array}$ \\
\hline Ptérygoödien Latéral (L) & $\begin{array}{l}\text { Muscle court, épais, en « } v \text { », à deux branches } \\
\text { ou faisceaux, inférieur condyloptérygoïdien et } \\
\text { supérieur disco-sphénoïdal. Le faisceau inférieur } \\
\text { est environ quatre fois plus épais que le faisceau } \\
\text { supérieur et contient approximativement } 80 \% \\
\text { des fibres musculaires }(15,10) \text {. }\end{array}$ & $\begin{array}{l}\text { Faisceau inférieur, oblique en bas, en dedans et en avant, tendu du col } \\
\text { du processus condylaire de la mandibule sur la fossette antéro-médiale } \\
\text { à la lame latérale du processus ptérygoïde et la face postéro-inférieure } \\
\text { de la tubérosité maxillaire. } \\
\text { Faisceau supérieur, à peu près horizontal, tendu de la capsule et du } \\
\text { bord antérieur du disque articulaire via la lame tendineuse pré-discale } \\
\text { à la face infra-temporale de la grande aile du sphénoïde et de la crête } \\
\text { sphénotemporale (limitrophe du T). }\end{array}$ \\
\hline Ptérygoïdien Médial (PM) & $\begin{array}{l}\text { Muscle épais, quadrilatère, situé médialement } \\
\text { par rapport au PL et tendu de la fosse ptérygoïde } \\
\text { à la face médiale de la branche montante et de } \\
\text { l'angle mandibulaire (15). }\end{array}$ & $\begin{array}{l}\text { Le faisceau principal descend obliquement en bas, en dehors et } \\
\text { en arrière, médialement au PL, et séparé de lui dans la fosse infra- } \\
\text { temporale par le fascia inter-ptérygoïdien. } \\
\text { Un faisceau aberrant déborde en avant et en dehors le PL, sur la } \\
\text { face latérale du processus pyramidal du palatin et la face maxillaire } \\
\text { attenante tubérositaire. }\end{array}$ \\
\hline
\end{tabular}

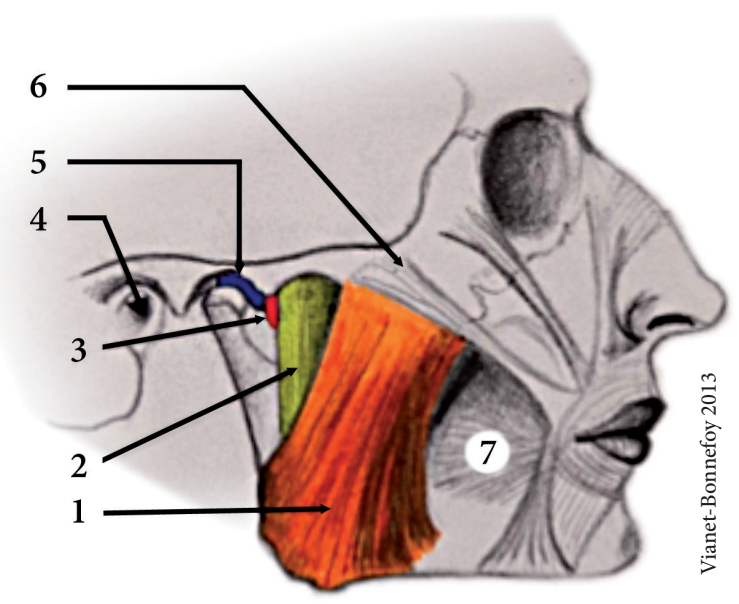

$\triangle$ Fig. 3 :

Sangle massétérique de profil.

1 : faisceau superficiel du M légèrement propulseur. 2 : faisceau moyen du M vertical. 3 : faisceau profond du M envoyant une expansion sur la lame tendineuse pré-discale. $4:$ méat acoustique externe. 5 : disque articulaire. 6 : muscle grand zygomatique (latéral au M). 7 : buccinateur (médial au M).

muscles, abaisseurs à distance de l'articulation, seront vus au chapitre traitant de l'anatomie fonctionnelle) : Idans le plan profond, le ptérygoïdien latéral (PL) abaisseur et le ptérygoïdien médial (PM) élévateur ; I dans le plan superficiel, le masséter (M) et le temporal (T) élévateurs.

Le masséter (M), élévateur superficiel, est facilement accessible à la palpation et intéressant par la saillie latérale de son bord antérieur qu'il provoque lors de sa contraction (Fig. 3). Il est impliqué dans les émotions

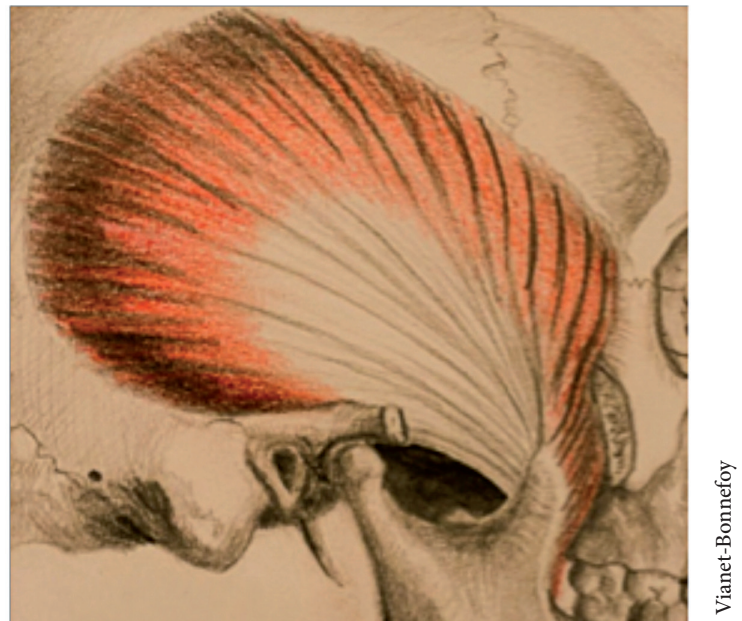

$\triangle$ Fig. 4 :

Muscle temporal en vue latérale droite.

La racine du zygoma a été retirée. Une expansion des fibres postérieures s'insère sur la lame tendineuse pré-discale médialement au masséter et latéralement au faisceau supérieur du ptérygoïdien latéral. Le rôle élévateur et rétropulseur est ici aisément compréhensible.

violentes telles que la colère, l'effort paroxystique, ou encore chez un certain type de patients dits " volontaires » qui ont tendance à serrer les mâchoires de façon consciente ou non (bruxisme). Le masséter est le plus puissant muscle du corps en rapport avec sa masse d'où son importance dans l'initiation des dysfonctions de l'ATM.

Le temporal (T) est également un muscle facilement palpable, responsable de la formation du processus coronoïde (coroné) (Fig. 4). 

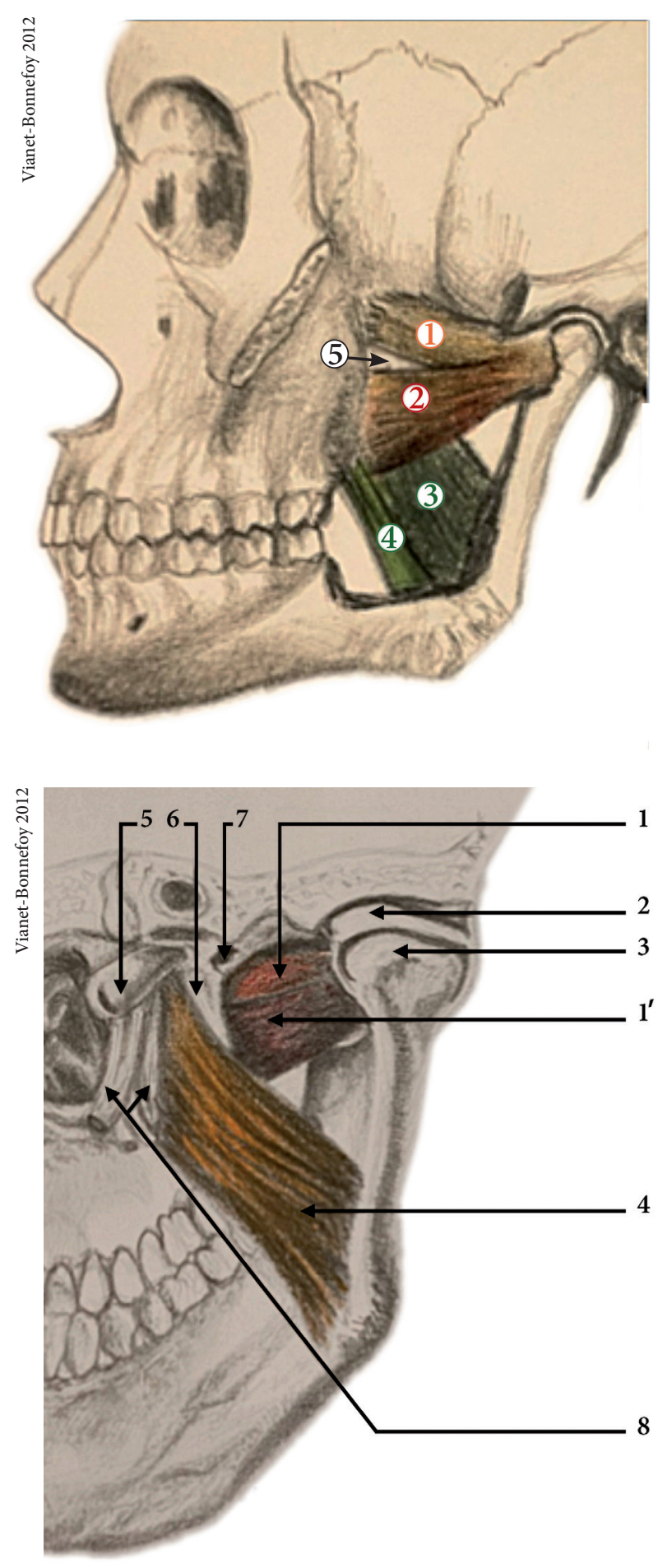

Le ptérygoïdien latéral (PL) est le seul muscle masticateur à avoir des rapports anatomiques étroits avec l'ATM : il est intra-articulaire puisque l'appareil discal a la même origine mésenchymateuse que le PL (Fig. 5 et 6). De par ses origines fibreuses, le disque correspond à l'extrémité postérieure du tendon d'insertion du PL $[2,17]$ qui coulisse entre les deux surfaces articulaires temporale et mandibulaire, ce qui implique nécessairement une grande importance dans la compréhension de l'anatomie fonctionnelle de cette articulation.

Le PM est un puissant muscle élévateur qui fait pendant à son homologue latéral et superficiel, le $M$, d'où son surnom de «masséter interne».
4 Fig. 5 :

Représentation classique du PL ou «modèle des deux chefs » [15].

1 : faisceau disco-sphénoïdal ou chef supérieur du ptérygoïdien latéral. 2 : faisceau condylo-ptérygoïdien ou chef inférieur du ptérygoïdien latéral. 3 : ptérygoïdien médial. 4 : faisceau aberrant tubérositaire du ptérygoïdien médial. 5 : espace triangulaire compris entre les deux faisceaux ou espace interfaisceaux dont l'importance varie en fonction du croisement de l'artère maxillaire qui s'appuie contre la face latérale du muscle avant de s'incliner en direction médiale et de s'enfoncer dans la fissure ptérygo-palatine. Le nerf buccal y passe également de médial en latéral, pouvant donner à chaque faisceau un rameau pénétrant par la face médiale du muscle. Il existe une grande variabilité dans l'innervation du PL (cf. chapitre 3 tableau innervation) mais les deux faisceaux ont toujours une innervation intrinsèque expliquant leurs fonctions différentes : tenseur du disque en fermeture pour contrer la force de rappel de la lame supérieure pour (1) et abaisseur, propulseur et diducteur controlatéral de la mandibule pour (2). Il existe une surface de glissement prolongeant l'espace interfaisceaux en arrière qui peut être prise à tort pour le disque mandibulaire à l'IRM.

4 Fig. 6 :

ATM et muscles ptérygoïdiens : vue postérieure, d’après Netter [12].

Les insertions du PM y sont visibles, à l'exception du faisceau aberrant tubérositaire montré dans la figure précédente. 1 : faisceau supérieur du PL. 1' : faisceau inférieur du PL. 2 : disque articulaire. 3 : processus condylaire mandibulaire. 4 : PM. 5 : partie cartilagineuse de la trompe d'Eustache. 6 : lame latérale du processus ptérygoïde. 7 : foramen oval livrant passage au V3. 8 : muscles élévateur et tenseur du voile du palais (sectionnés pour plus de lisibilité du dessin). Son action diductrice controlatérale est bien visible sur ce schéma ainsi que, sur le schéma précédent, son action propulsive.

\section{ARTHROLOGIE}

LATM est une articulation bi-condylaire à mobilité importante (diarthrose) à disque interposé : elle est considérée comme la plus mobile de toutes les articulations humaines (Fig. 7, 8 et 10). Or, plus une articulation est mobile et moins elle est stable et peut se luxer [9]. La présence de structures cartilagineuses est la conséquence de mouvements précoces résultant de l'effet des muscles du système manducateur : succion, déglutition puis mastication et phonation. Sans leur action, cette articulation ne serait qu'une suture cranio-faciale. La fosse mandibulaire se creuse grâce au phénomène de la mastication intervenant lors de la mise en place sur l'arcade des premières molaires mandibulaires. Cette morphologie se confirme avec la mise en place des dents de 12 ans. Un lien très étroit de réciprocité existe donc entre l'ATM et les régions molaires maxillo-mandibulaires. L'ATM sert de calage postérieur pour la mastication et les secteurs molaires de calage postérieur pour l'ATM.

«L'appareil disco-ligamentaire $[8,6,10]$ n'est qu'un des éléments de la triade comprenant la capsule articulaire 
1

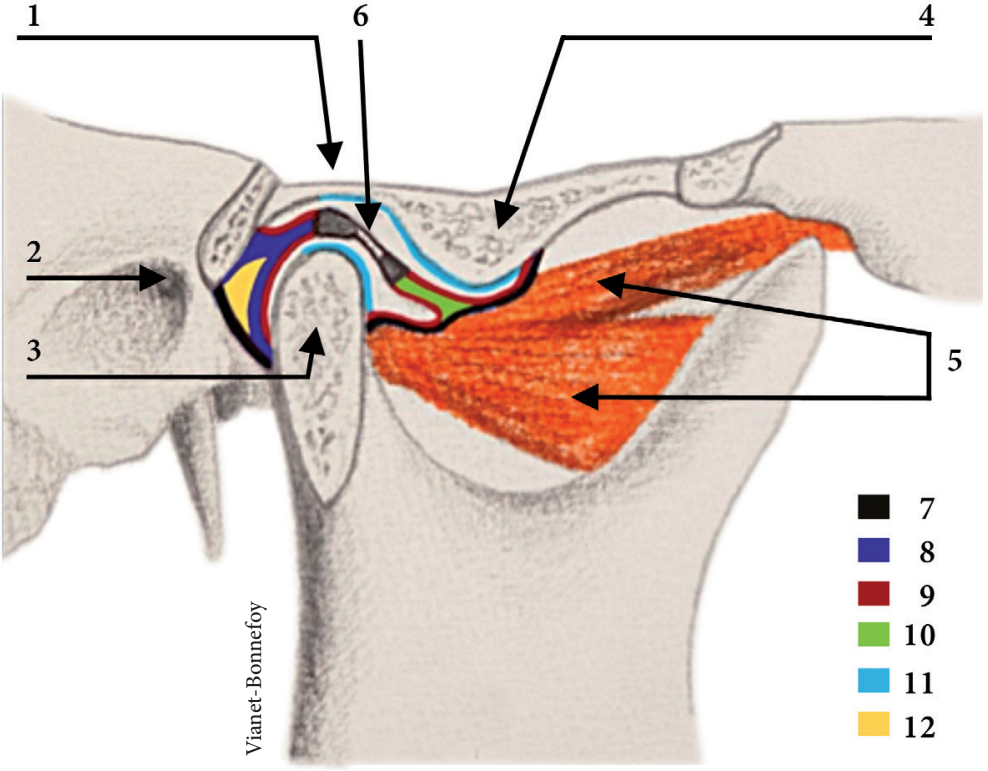

4 Fig. 7 :

Coupe sagittale de l'ATM droite en bouche fermée : le PL est solidaire du complexe discal par son faisceau supérieur. Les parois latérales et médiales de la capsule n'y sont pas visibles car en dehors du plan de coupe. Les fibres massétériques et temporales, qui sattachent latéralement sur la lame tendineuse prédiscale et qui masquent une bonne partie de la fosse infratemporale ainsi que l'incisure sigmoïde ont été réséquées pour plus de clarté du schéma.

1 : fosse mandibulaire. 2 : méat acoustique externe.

3 : processus condylaire mandibulaire. 4 : tubercule articulaire. 5 : ptérygoïdien latéral présentant ses deux faisceaux. 6 : disque articulaire constitué d'un bourrelet postérieur et d'un bourrelet antérieur séparé par une zone centrale amincie et solidarisé au processus condylaire par des ailerons discaux à ses pôles latéral et médial. $7:$ capsule articulaire. $8:$ lame bilaminaire constituée par la réunion du frein temporo-discal ou lame supérieure et du frein discal postérieur ou lame inférieure. 9 : membrane synoviale. 10 : ligament discal antérieur ou lame tendineuse prédiscale. 11 : zone articulaire fibrocartilagineuse temporale et mandibulaire. 12 : zone interlaminaire constituant un petit espace, postérieure au sein de l'ATM délimitée par 7 et 8.

4 Fig. 8 :

Coupe sagittale de l'ATM en bouche ouverte : le disque est tendu et stabilisé entre les deux surfaces convexes condylo-temporales, à l’aplomb de la partie la plus déclive du tubercule articulaire. 1 : méat acoustique externe. 2 : lame bilaminaire composée de la lame supérieure ou frein temporo-discal et de la lame inférieure ou frein discal postérieur. 3 : fosse mandibulaire du temporal. 4 : disque ayant tendance à sétirer lors de l'ouverture buccale. 5 : tubercule articulaire du temporal. 6 : lame tendineuse prédiscale se confondant avec la capsule articulaire et les lames tendineuses du faisceau supérieur du PL. 7 : zone interlaminaire contenant le coussinet vasculaire de Zenker qui augmente de volume allant jusqu'à combler l'espace libre laissé derrière lui par le processus condylaire lors du mouvement d'abaissement de la mandibule. 8 : capsule articulaire surtout identifiable en arrière sur les coupes sagittales de l'articulation. 9 : processus condylaire mandibulaire. 10 : PM avec le corps du muscle (vert clair) et le faisceau aberrant rétrotubérositaire (vert foncé). 11 : PL : faisceau supérieur et inférieur.

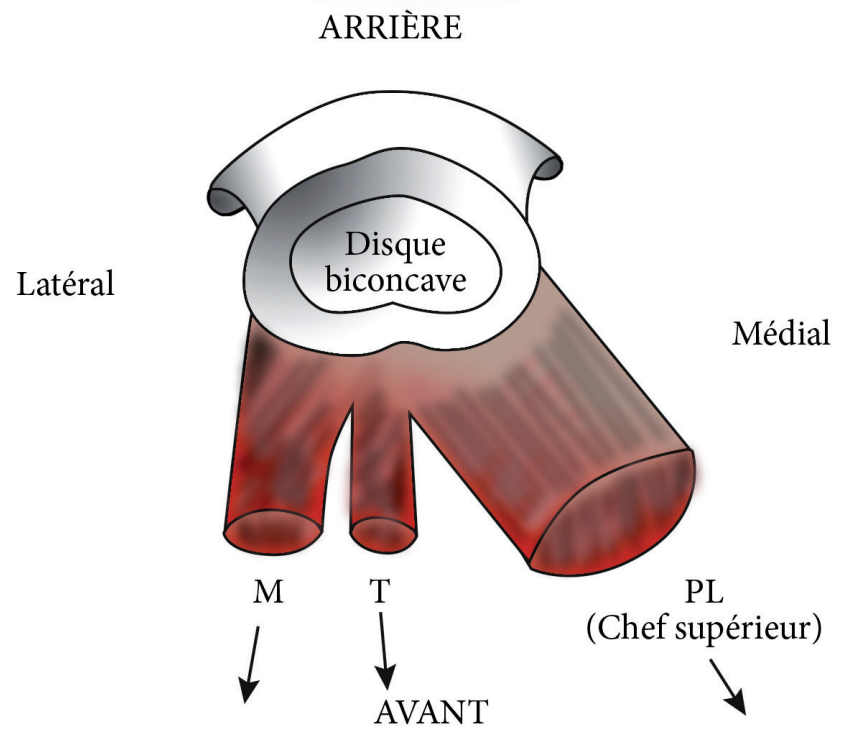

$\triangleleft$ Fig. 9:

Action sur le disque de l'ATM, en vue craniale, des muscles s'insérant sur le bord antérieur capsulaire, ce dernier se confondant en réalité avec la lame tendineuse prédiscale. $\mathrm{M}$ : masséter. T : temporal. PL : ptérygoïdien latéral. De médial en latéral, sur le bord antérieur de l'appareil disco-capsulaire, on individualise successivement le faisceau supérieur du PL, une expansion du temporal et enfin une expansion postérieure de la couche profonde du faisceau profond du masséter. Ces trois muscles agissent sur le disque par guidage tridirectionnel. Ils agissent sur la position du disque non seulement dans le sens sagittal mais aussi dans le sens transversal. En chirurgie de l'ATM, toute interposition de tissu devra respecter cette action mobilisatrice afin d'obéir à l'aspect fonctionnel et d'éviter l'ankylose (Delaire, 1983). 


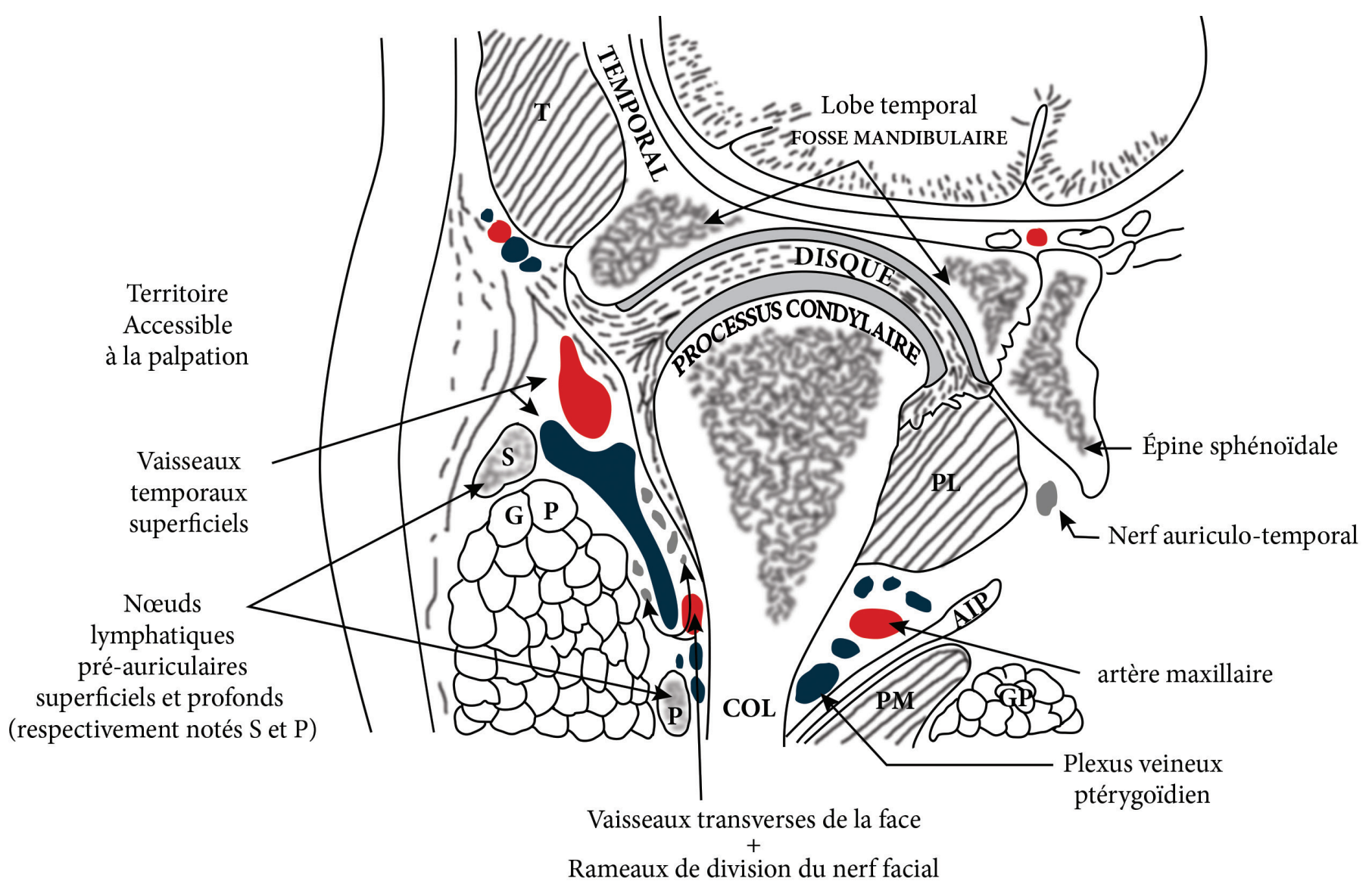

$\triangle$ Fig. 10 :

Coupe coronale de l'ATM avec hémisection du processus condylaire mandibulaire, d'après Maillot et Kahn 2004 [13].

$\mathrm{T}$ : muscle temporal. PM : muscle ptérygoïdien médial. PL : muscle ptérygoïdien latéral. GP : glande parotide. AIP : aponévrose interptérygoïdienne.

[7] et le ptérygoïdien latéral [5], muscle puissant dont la contraction a essentiellement pour effet de propulser la mandibule et le disque, qui sont solidarisés par les ailerons discaux, formant un complexe condylo-disco-musculaire indissociable » (citation in extenso de [11]). Le disque est classiquement solidaire du processus condylaire et du faisceau supérieur du PL dont il forme le tendon d'insertion (se prolongeant antérieurement par la lame tendineuse prédiscale et la capsule qui sont solidaires du disque et postérieurement par la lame bilaminaire) mais aussi des expansions du $\mathrm{M}$ et du $\mathrm{T}$, ce qui est notable dans la compréhension des dysfonctions de l'ATM [2] (Fig. 9).

Classiquement, le disque présente la forme d'une lentille biconcave, ou d'un "globule rouge " présentant un bourrelet périphérique et un centre aminci, nécessaire au bon fonctionnement de l'ATM du fait de la convexité des surfaces articulaires en présence. Le bourrelet périphérique est deux fois plus épais en arrière qu’en avant : le disque présente un bourrelet postérieur et un bourrelet antérieur.
Le disque, fibrocartilagineux (cellules de type cartilagineux dispersées entre les fibres de collagènes type III dans les zones de stress mécanique), est par nature susceptible de remodelage lors des kinésithérapies dites « d'éviction discale ». Le but de cette mécanothérapie est de créer un "néodisque » [2] en poussant le disque vers lavant et en le remodelant par frottement entre les deux surfaces articulaires temporo-mandibulaires avec des mouvements de diduction controlatéraux et de propulsion mandibulaire afin de lever le blocage et de récupérer in fine de l'amplitude à l'ouverture buccale. Le bourrelet postérieur protège le fragile plafond de la fosse mandibulaire en jouant le rôle de tampon. Le bourrelet antérieur se situe en occlusion en avant du processus condylaire et en arrière de la partie la plus inférieure du tubercule articulaire. Le disque est donc oblique en bas et en avant. Il est constitué de fibres de collagène orientées dans les trois directions de l'espace pour résister aux forces exercées sur lui par les trois muscles : PL, T et M (résistance à la compression entre 180 et $220 \mathrm{~kg} \mathrm{par} \mathrm{cm}^{2}$ ). Il nest pas innervé et ne peut être impliqué directement dans la symptomatologie. 
Le disque cloisonne l'articulation en deux compartiments [12] :

I supérieur disco-temporal de volume égal en moyenne à $1,2 \mathrm{~mL}$;

ı inférieur disco-mandibulaire ou condylo-discal de volume égal à $0,9 \mathrm{~mL}$.

En partie rétro-discale de l'ATM, la zone bilaminaire comprend en haut la lame supérieure (ou frein temporo-discal) élastique et en bas la lame inférieure (ou frein condylo-discal) solide et collagénique. Elle enchâsse la zone interlaminaire qui contient le coussin vasculaire de Zenker. Elle possède un rôle amortisseur de pressions, est la source dépanchement articulaires et est richement innervée par des fibres sensitives du nerf auriculo-temporal, nerf principal de l'ATM et branche de division $\mathrm{du}$ tronc terminal postérieur du nerf mandibulaire (V3 [2]) (Fig. 10 et 11). Ce nerf participe également à l'innervation sensitive de la fosse temporale et de loreille externe. Ceci explique que les patients qui présentent un dysfonctionnement de l'ATM (par rétroposition du processus condylaire provoquant une compression de la zone bilaminaire par exemple) présentent également souvent des otalgies et/ou une céphalée temporale [17]. En cas de spasme du faisceau supérieur du PL, la zone bilaminaire peut se distendre par traction sur le ressort modélisant la lame supérieure. Enfin la capsule possède différents récepteurs sensitifs, notamment nociceptifs (douleurs possibles de type capsulite rétractile avec compression de la zone bilaminaire).
L'innervation motrice de l'ATM est sous la dépendance du V3 qui donne en particulier un tronc commun pour les muscles du PM, du tenseur du voile et du tenseur du tympan (Fig. 11). Cela peut expliquer les signes otologiques parfois observés dans les syndromes dysfonctionnels de l'ATM sans recourir à une quelconque perméabilité de la scissure pétro-tympano-squameuse (laquelle serait alors visible radiologiquement), incriminée dans quelques cas [16].

$N B$ : tous les muscles masticateurs sont innervés par le V3 à deux exceptions près : le ventre postérieur du digastrique innervé par le nerffacial et le génio-hyoïdien innervé par le nerf hypoglosse. Le nerf $d u$ PL donne un rameau supérieur pour le faisceau supérieur et un rameau inférieur pour le faisceau inférieur, ce qui explique leurs rôles fonctionnels différents (cf. figure 5, représentation classique du PL).

Comme l'innervation sensitive de l'ATM dépend également du nerf mandibulaire, ceci explique le cercle vicieux qui s'installe en cas de dysfonctionnement de cette articulation [17] : la douleur entretient le spasme des muscles masticateurs qui, lui-même, accentue celle-ci. Il est donc nécessaire de déconditionner les désordres neuro-musculaires par tous les moyens thérapeutiques en notre possession : plan de libération occlusal, kinésithérapie, adjuvants médicamenteux. Les injections de toxine botulique sont utilisées en dernier recours [4].

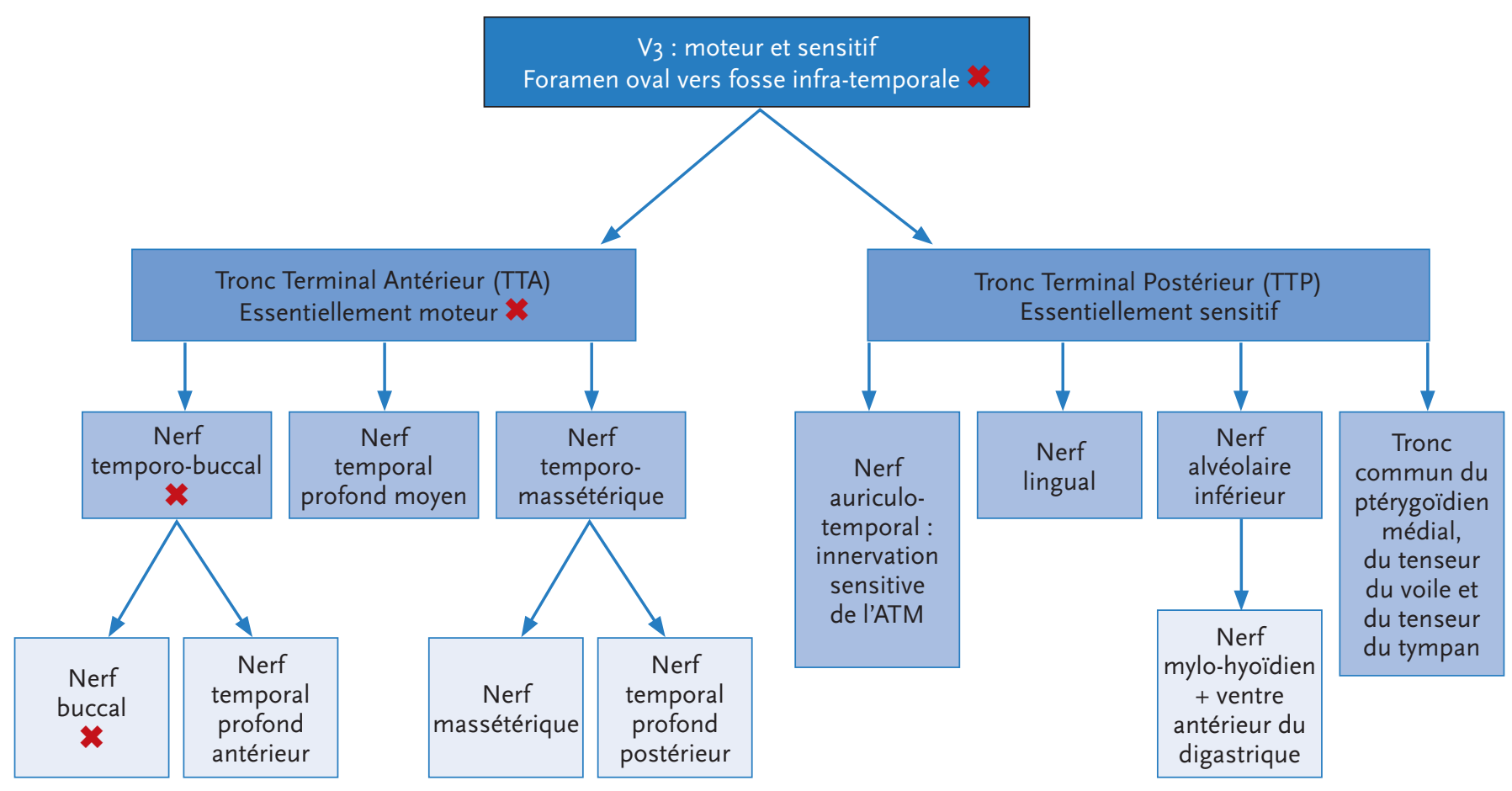

Fig. 11 :

Innervation du V3, nerf contrôlant la sensibilité et la motricité de l’ATM. Le nerf du ptérygoïdien latéral n'a pas été mis intentionnellement car d'une grande variabilité. Il peut émerger directement du V3, de son TTA, du temporo-buccal et même du buccal. Ces nerfs sont marqués d'une croix. 


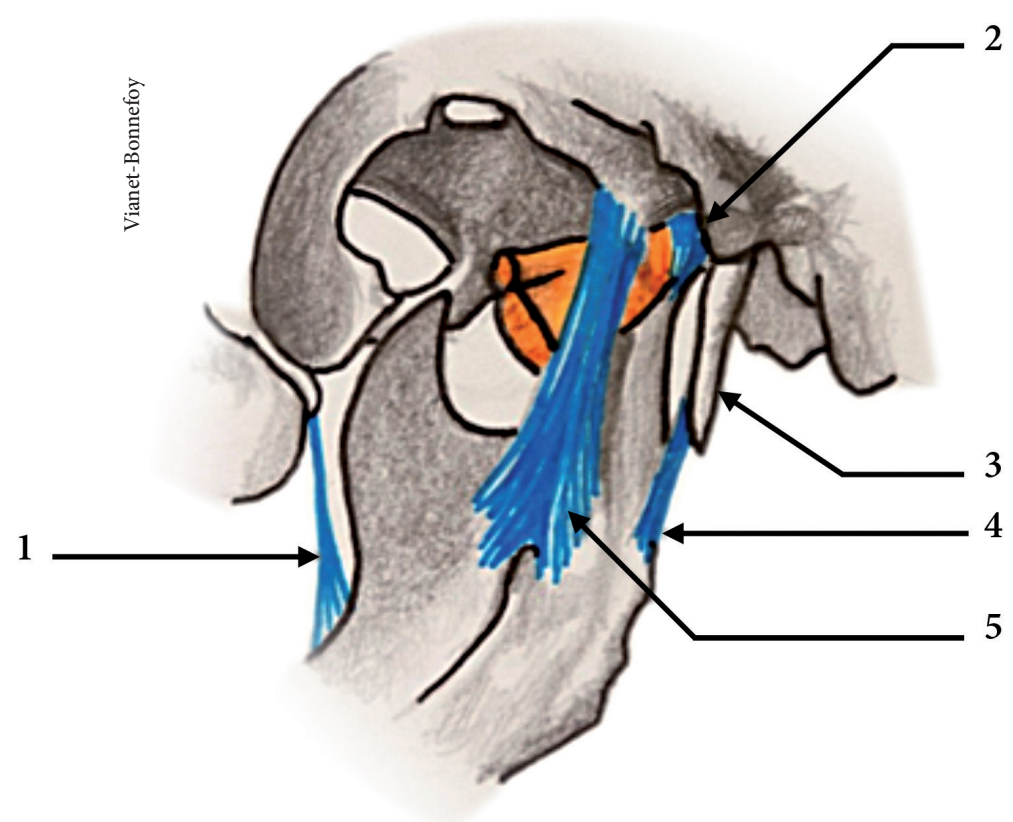

$\triangleleft$ Fig. 12 :

Ligaments suspenseurs de l'ATM en vue médiale.

1 : raphé ptérygo-mandibulaire. 2 : capsule et ligament médial dans le prolongement du PL dont les deux faisceaux sont représentés ici en orange ; 3 : processus styloïde du temporal.

4 : ligament stylo-mandibulaire. 5 : ligament sphéno-mandibulaire dans le prolongement de l'aponévrose inter-ptérygoïdienne (AIP) avec en dessous lémergence du nerf mylo-hyoïdien. Le PL est visible (couleur orange) par ses insertions discomandibulaires.

Les ligaments sont classés en ligaments intrinsèques et extrinsèques. Ils sont dits suspenseurs de l'articulation (Fig. 12). Les ligaments intrinsèques sont constitués : I des ligaments discaux collatéraux ou ailerons peu ou pas élastiques ;

\du ligament latéral qui est un épais ligament recouvrant la face latérale de la capsule articulaire et qui limite anatomiquement les déplacements en latéralité, rétropulsion et abaissement du processus condylaire ; ı du ligament médial de moindre importance que le ligament latéral.

Les ligaments extrinsèques sont constitués : Idu ligament stylo-mandibulaire, simple bandelette fibreuse formée par un épaississement du fascia cervical profond allant du processus styloïde au bord postérieur de l'angle et du ramus mandibulaire. Il limite le mouvement d'abaissement en propulsion de la mandibule ;

Idu ligament sphéno-mandibulaire, reliquat du cartilage de Meckel, qui va de l'épine du sphénoïde à la lingula et l'anti-lingula mandibulaire constituant un important moyen de contention de l'ATM ;

Idu ligament ou raphé ptérygo-mandibulaire sans importance fonctionnelle pour l'ATM.

\section{ANATOMIE FONCTIONNELLE}

\section{Généralités}

Les surfaces articulaires mandibulaires et temporales sont soumises au frottement du tendon du muscle ptérygoïdien latéral qui constitue l'appareil discal dont la structure fibreuse et riche en collagène lui permet ainsi de jouer un rôle d'interposition et de glissement indis- pensable au bon fonctionnement de la biomécanique articulaire [2]. Des études de modélisation biomécanique au sein de l'ATM montrent qu'en l'absence de disque, les contraintes exercées sur le processus condylaire sont beaucoup plus importantes et bien moins réparties, protégeant ainsi les surfaces articulaires de l'usure [1]. Le disque stabilise également l'articulation, la convexité du processus condylaire ne s'accordant pas avec celle du tubercule articulaire.

LATM permet locclusion, les bases maxillo-mandibulaire s'articulant par engrènement de l’articulé dentaire. Si l'anatomie descriptive de l'appareil manducateur est maintenant globalement comprise, c'est au niveau fonctionnel que les difficultés apparaissent. Cliniquement, les douleurs présentes lors des algies de l'appareil manducateur (ADAM) ou des syndromes algo-dysfonctionnels de l'appareil manducateur (SADAM) « témoignent d'une réelle maladie de l'articulation » [14]. On a tenté de les classifier en douleurs articulaires induites par compression de la zone bilaminaire ou par atteinte de l'intégrité anatomique (arthropathies, destructions par maladies de systèmes, tumorales, etc.) et extra-articulaires d'origine musculaire essentiellement spastiques. Une convergence pluridisciplinaire s'est révélée fructueuse dans la compréhension de l'anatomie fonctionnelle de l'ATM en cherchant à la relier à l'aspect clinique qui conditionne l'approche diagnostique et thérapeutique $[3,4]$.

\section{Biomécanique articulaire et cinétique mandibulaire}

Le fonctionnement d'une ATM ne dépend pas seulement de ses propres conditions fonctionnelles mais aussi du fonctionnement de l'ATM contra-latérale. C'est pourquoi en cas de déficit d'amplitude à l'ouverture 
d'une articulation, l'ATM contralatérale va essayer de le compenser par une amplitude plus importante que la normale (subluxation compensatrice).

Lors de louverture buccale, on estime généralement que le processus condylaire effectue d'abord un mouvement de rotation pure dans le compartiment inférieur, jusqu'à $2 \mathrm{~cm}$ dans le plan sagittal, en tournant contre la face inférieure du disque puis un mouvement de translation en avant et en bas dans le compartiment supérieur guidé par l'orientation de ce même disque sur le tubercule articulaire du temporal. En réalité, le mouvement est progressif en fonction du degré d'ouverture buccale et ainsi rotation et translation peuvent s'associer en formant une « rototranslation ». Lors des mouvements amples d'ouverture buccale, l'axe charnière de rotation mandibulaire n'est pas bi-condylaire (comme il l'est pour les mouvements proches de la position de repos mandibulaire) mais situé au niveau de la lingula et de l'entrée du canal mandibulaire. Normalement, les deux processus condylaires et coronoïdes ont des longueurs équivalentes : toute variation des longueurs de ces processus modifiera l'ampleur et l'équilibre des mouvements produits par les muscles qui s'insèrent sur eux [6].

Lors des mouvements de diduction, le processus condylaire du côté travaillant effectue une légère translation en dehors, appelée mouvement de Bennett, tandis que le processus condylaire controlatéral se déplace légèrement en avant, en dedans et en bas selon un angle avec le plan sagittal en projection sur un plan horizontal nommé angle de Bennett (Fig. 13). Les deux processus condylaires, côté travaillant et non-travaillant, entraînent chacun leur disque respectif qui leur sont solidaires dans leurs déplacements [5].

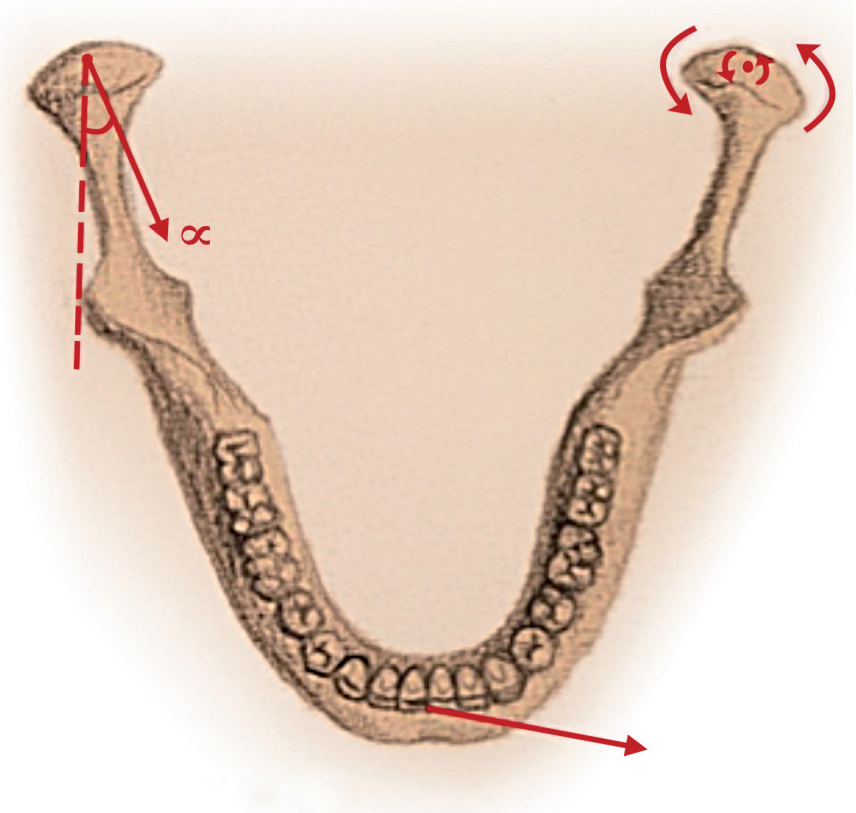

Lors des mouvements de propulsion, la mandibule se dirige en avant et en bas par translation du complexe inférieur condylo-discal le long de la surface postérieure du tubercule articulaire. La rétropulsion est un mouvement physiologiquement assez limité (faisceaux postérieurs du T), le processus condylaire et son disque devant obligatoirement comprimer la zone bilaminaire avant de buter sur la partie postérieure de la fosse mandibulaire et le tubercule zygomatique postérieur. Le processus condylaire vient à l'aplomb du tubercule articulaire du temporal en ouverture maximale physiologique, c'est-à-dire qu'il atteint (ou dépasse de peu) le point le plus déclive du tubercule articulaire de l'os temporal [3].

Si un obstacle existe sur le trajet d'un processus condylaire, lorsque survient un épisode de luxation non réductible du disque, le processus condylaire ne parvient pas, par définition, à l'aplomb du tubercule articulaire et louverture buccale s'en trouve réduite de ce côté. Il reste alors bloqué dans sa "pente condylienne », c'est-à-dire dans la déclivité antérieure de la fosse mandibulaire, en arrière du sommet du tubercule articulaire du temporal. La rétroposition condylaire comprime la zone bilaminaire et exacerbe les douleurs dans le territoire d'innervation du nerf auriculo-temporal.

Si le processus condylaire va au-delà de l'aplomb, on parle de luxation condylo-temporale : il peut alors se produire un blocage en bouche ouverte. Si un processus condylaire ne parvient pas à l'aplomb, le processus condylaire controlatéral effectue alors une subluxation compensatrice.

Les forces engendrées par les muscles élévateurs lors de la déglutition d'aliments solides s'exercent nor-

4 Fig. 13:

Mouvement et angle alpha de Bennett.

Du côté travaillant, soit celui du mouvement, le déplacement latéral appliqué ici classiquement sur le dentalé, ou point intericisif mandibulaire, est appelé mouvement de Bennett. Le processus condylaire est dit pivotant. Le processus condylaire opposé, dit orbitant, se déplace selon l'angle de Bennett de valeur moyenne d'environ $12^{\circ}$. 


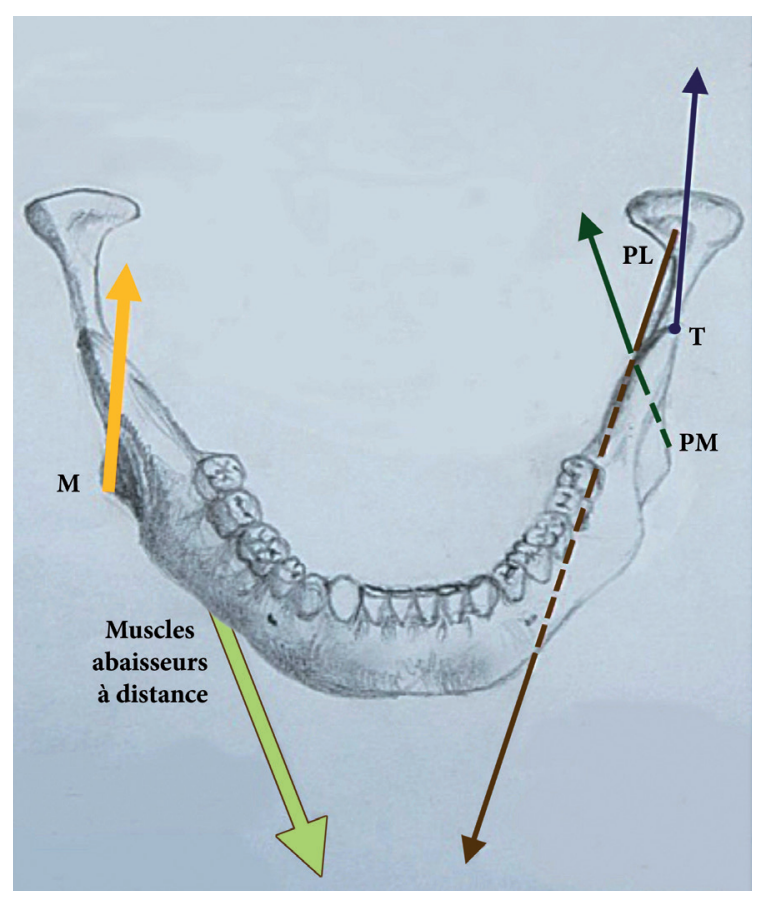

malement sous la forme de contacts occlusaux en intercuspidation maximale (Fig. 14, 15 et 16) mais ces contacts ne sont pas une généralité en ce qui concerne les aliments liquides ou simplement le fait d'avaler sa salive où la position de la mandibule peut rester voisine de sa position de repos, ce qui implique nécessairement alors un déplacement faible du processus condylaire dans la fosse mandibulaire de l'os temporal et proche de l'équilibre musculaire : l'axe charnière est bicondylaire $\triangleleft$ Fig. 14 :

Action des muscles du système manducateur sur la mandibule, conférence AEOS Dichamp 2009, résumée in [2] et basée sur lobservation clinique des fractures de la mandibule. $\mathrm{M}$ : action en haut et en avant soit élévation et propulsion par ses fibres superficielles. $\mathrm{T}$ : action en haut et en arrière soit élévation par sa partie antérieure et moyenne et rétropulsion par sa partie postérieure. PM : action en haut, en avant et en dedans soit élévation et propulsion si action bilatérale ou diduction controlatérale si action unilatérale.

PL par son faisceau inférieur : action en bas, en avant et en dedans soit abaissement et propulsion si action bilatérale et diduction controlatérale.

Les deux muscles droit et gauche peuvent se contracter séparément donnant toute une enveloppe de mouvements assimilable à une circumduction. Muscles abaisseurs mandibulaires à distance de l'articulation comme le digastrique, le mylo-hyoïdien et le génio-hyoïdien (synergie avec le platysma) : action en bas, en dedans et en arrière soit abaissement et rétropulsion si action bilatérale et diduction si action unilatérale. Le ventre antérieur du digastrique est seul considéré comme abaisseur. Le ventre postérieur participe à la stabilisation de l'os hyoïde en coopération avec les muscles sous-hyoïdiens. Pour agir sur la mandibule, les muscles abaisseurs ont besoin que l'os hyoïde soit stabilisé afin de servir d'appui.

avec légère rotation des processus condylaires sur leur axe. Toutefois, les forces axiales exercées sur les dents par les muscles élévateurs sont importantes en cas de serrage, variant entre 60 et $80 \mathrm{~N}$.

C'est durant la mastication, surtout d'aliments solides et épais, que la biomécanique musculaire est la plus mise à contribution avec le risque de spasmes musculaires lors de l'intervention de tous les muscles élévateurs, diducteurs et abaisseurs. En outre, louverture
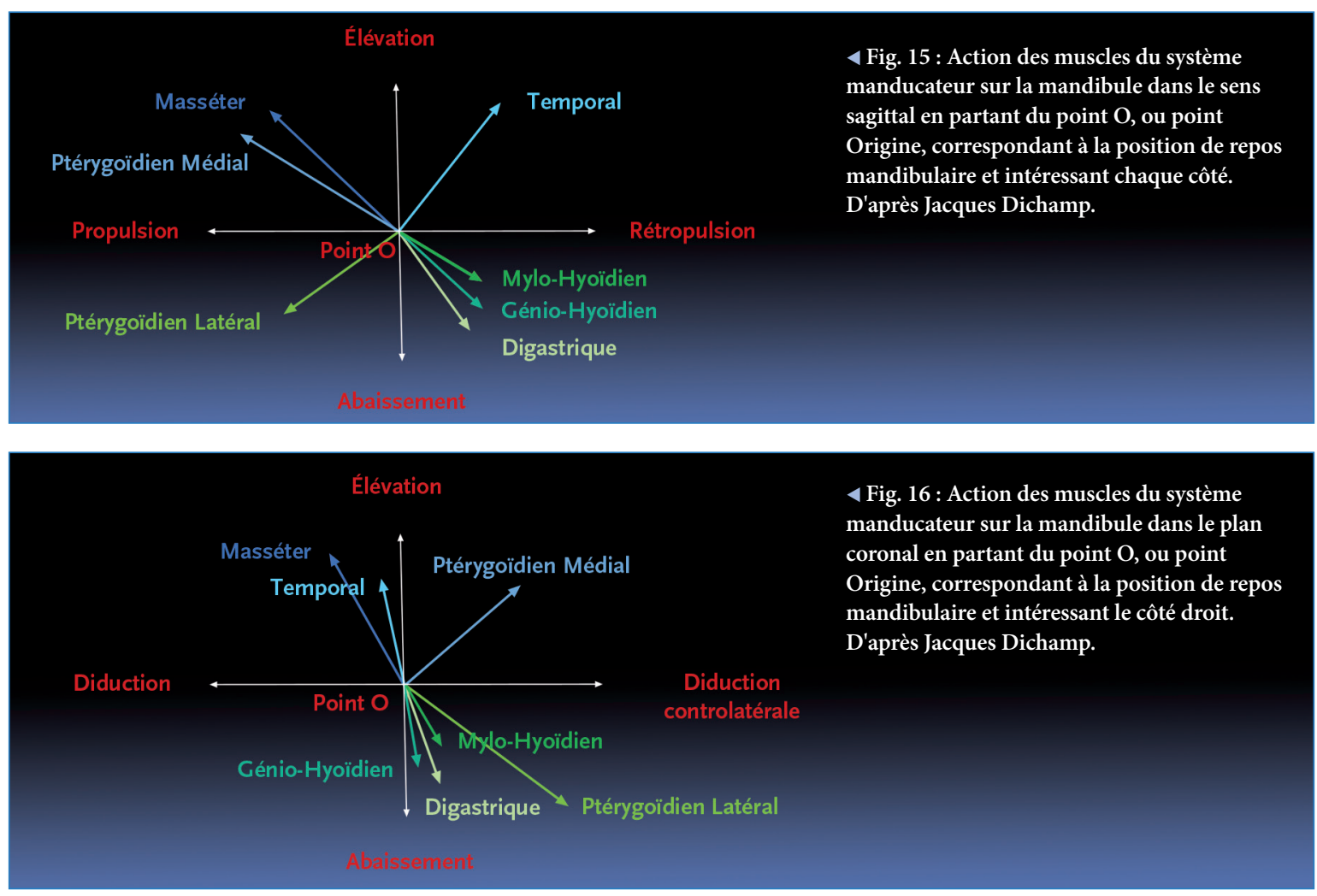
buccale forcée lors de la mise en bouche de l'aliment exige pour son écrasement efficace une pression importante produite par les élévateurs avec mouvements excentrés pour broyer le bol alimentaire au niveau des secteurs postérieurs molaires associés à une mastication unilatérale alternée.

En ouverture, le disque est essentiellement soumis à des contraintes de cisaillement. Ces dernières augmentent jusquà la moitié de l'amplitude maximale physiologique, se relâchent lorsque le disque passe avec succès le tubercule articulaire puis augmentent à nouveau ensuite avec une prédominance des contraintes verticales de compression $[1,2]$. Lassociation cisaillement-compression produit une usure du disque articulaire avec le temps. Chez les sujets âgés, on note des dysfonctions de larticulation dans $25 \%$ des cas [9]. Cela peut expliquer que, dans les dysfonctions sévères lors du bruxisme, la partie latérale ou centro-discale est perforée et les contraintes excessives induites par les muscles élévateurs peuvent aller jusquà provoquer la désagrégation du disque. Laction des muscles du système manducateur lors des parafonctions possède une certaine variabilité individuelle comme dans le bruxisme centré, par serrage des élévateurs, ou excentré, avec action concomitante des ptérygoïdiens [4].

\section{Cinétique mandibulaire et occlusion}

Les muscles, et non le système dentaire, déterminent de façon fonctionnelle, comme la traumatologie le démontre, la position de la mandibule et du disque articulaire pendant toute la cinématique 3D dans l'enveloppe de tous les mouvements possibles.

La position de repos de la mandibule est déterminée par un équilibre musculaire entre les agonistes et les antagonistes déterminant un espace libre dento-dentaire physiologique denviron 3 à $4 \mathrm{~mm}$. Le processus condylaire est centré dans la fosse mandibulaire de los temporal d'où le terme de relation myo-centrée.
Locclusion d'intercuspidation maximale (OIM) est déterminée par l'articulé dentaire ou engrènement dentaire et est obtenue lorsqu'on demande au patient de fermer la bouche, c'est-à-dire d'activer ses muscles élévateurs, et de placer ses dents dans leur position habituelle. Le processus condylaire recule dans la fosse mandibulaire par rapport à la position de repos et comprime un peu plus la zone bilaminaire.

Locclusion dite « de convenance » correspond cliniquement à la relation d'intercuspidation maximale, propre à l'évolution du système dentaire de chaque individu, le système manducateur ayant naturellement tendance en fermeture à tendre vers la dimension verticale docclusion (DVO) la plus faible. Les muscles élévateurs de la mandibule exercent pleinement leur puissance lors de la mise en contact des dents maxillo-mandibulaires et particulièrement lors de la mastication ou de locclusion active, lorsque le patient, ayant atteint sa position d'OIM, serre franchement les dents (possibilité de douleur en cas de rétroposition du processus condylaire puisque c'est la position fonctionnellement la plus postérieure en occlusion).

Le système alvéolo-dentaire sert surtout de butée d'enfoncement et d'appui proprioceptif en occlusion (récepteurs desmodontaux palliant labsence de capteurs de pression au niveau du disque articulaire) permettant aux muscles élévateurs d'exercer leur puissance de broyage sur le bol alimentaire mais dexacerber conjointement les contraintes mécaniques sur le disque, lui-même amortisseur et répartiteur de contraintes sur les surfaces articulaires attenantes. À ce jeu, c'est l'usure du disque contre celle du système dentaire. Le bruxisme [4] provoque une abrasion de l'anatomie occlusale dentaire sans nécessairement provoquer de SADAM. (Fig. 17)

La fonction crée la forme : les molaires ont été façonnées au cours de lévolution dans la zone d'influence du complexe élévateur temporo-massétérique et servent à faire passer la puissance de contraction par broyage

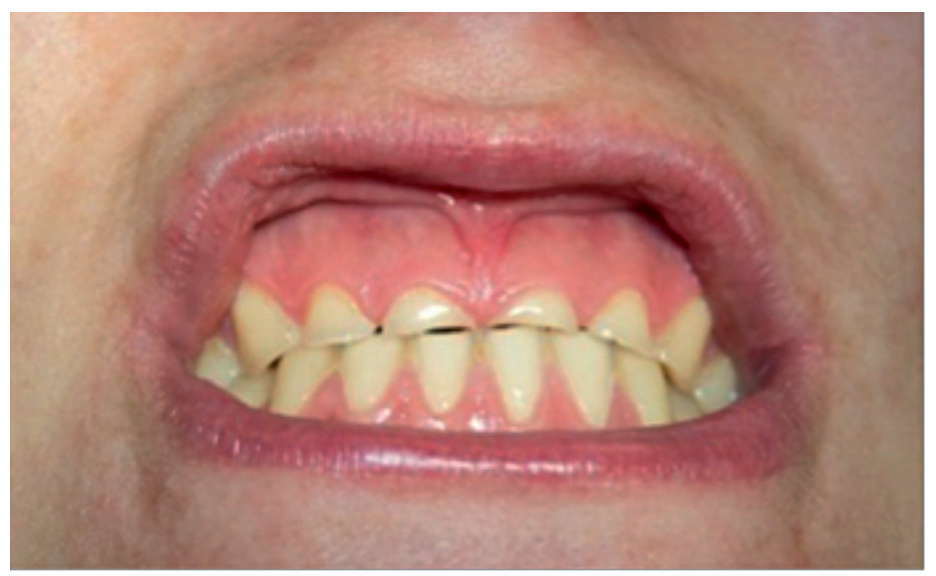

4 Fig. 17:

Abrasions dentaires chez un jeune adulte atteint de parafonctions avec bruxisme excentré. L'usure des dents, surtout maxillaires, en secteur antérieur par mouvements excentrés a conduit à une forte demande esthétique sans retentissement algique. Patient vu lors de la consultation sur les dysfonctions de l'ATM des Dr Chikhani et Dichamp du service de stomatologie de l'hôpital européen Georges Pompidou. 

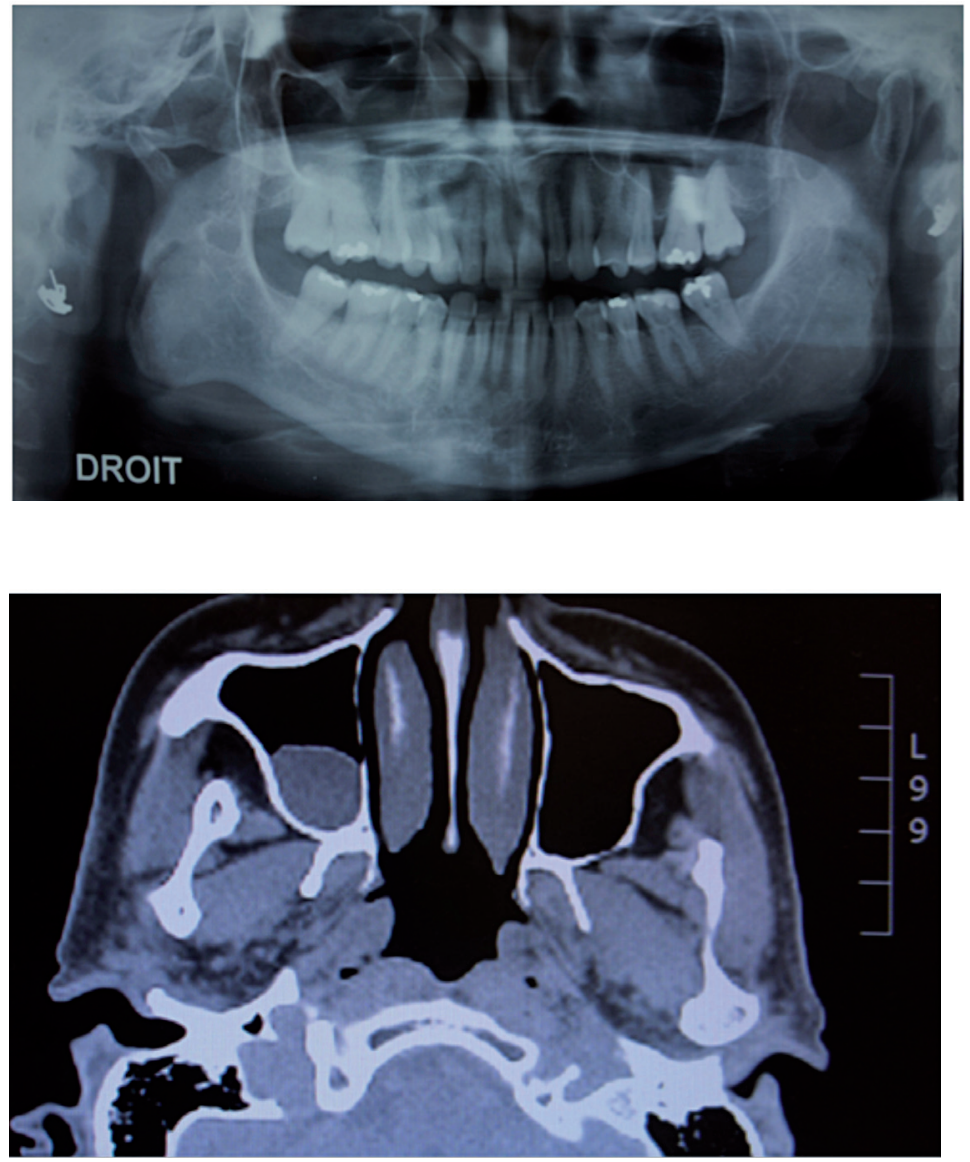

$\triangleleft$ Fig. 18:

Ce cliché panoramique montre l'intérêt de cet examen complémentaire en première intention avec un processus condylaire anatomiquement normal gauche et un processus microcondylaire droit avec asymétrie faciale. Cette anomalie anatomique particulièrement marquée n’a aucun retentissement fonctionnel, la patiente se plaint du problème esthétique posé par son asymétrie faciale depuis son plus jeune âge.

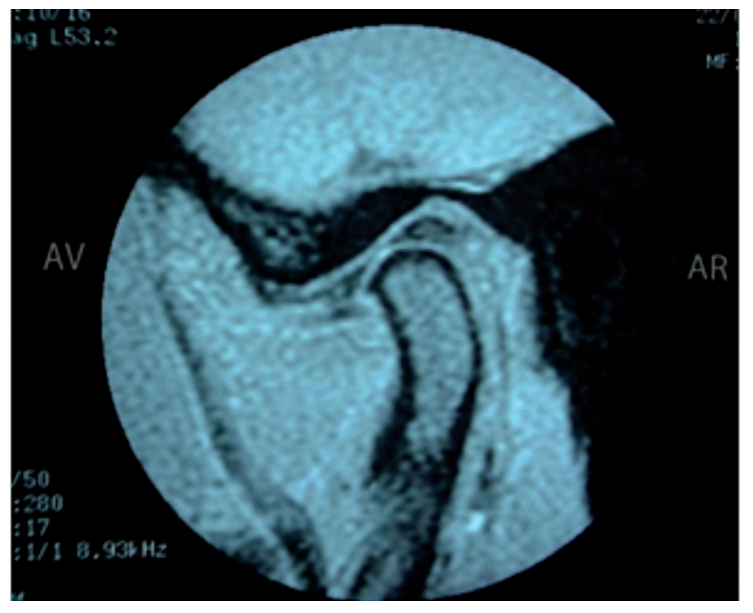

$\triangle$ Fig. 20 :

IRM bouche fermée avec processus condylaire en place dans la fosse mandibulaire. Le disque est ici incliné dans le plan sagittal selon la pente condylienne présentant un angle de près de $45^{\circ}$ avec l'horizontale (iconographie Jacques Dichamp).

sur le bol alimentaire à la manière d'une meule, ce que révèle leur étymologie. Le masséter étend sa zone d'influence en secteur postérieur à peu près jusquà la première molaire. Labsence de calage dentaire par perte des molaires entraîne une instabilité mécanique en favorisant une mastication dysfonctionnelle (propulsion et élévation conjointe par les deux PMs $\varangle$ Fig. 19:

Coupe axiale TDM CT-SCAN présentant un microprocessus condylaire gauche et un processus condylaire morphologiquement normal à droite. Les grands axes de chacun des condyles se croisent en un point situé en dehors du foramen magnum et déporté du côté présentant l'anomalie anatomique. La capsule et le disque articulaire ne sont pas visibles en coupe sagittale et constituent la limite de cet examen dans les cas de luxations discales. Des progrès revendiqués par les fabricants sur les scanners de toute dernière génération demandent à être confirmés. L'excellente visibilité des anomalies anatomiques et des destructions osseuses (géodes sous-chondrales, érosions, ostéonécroses) en font un examen de prédilection.

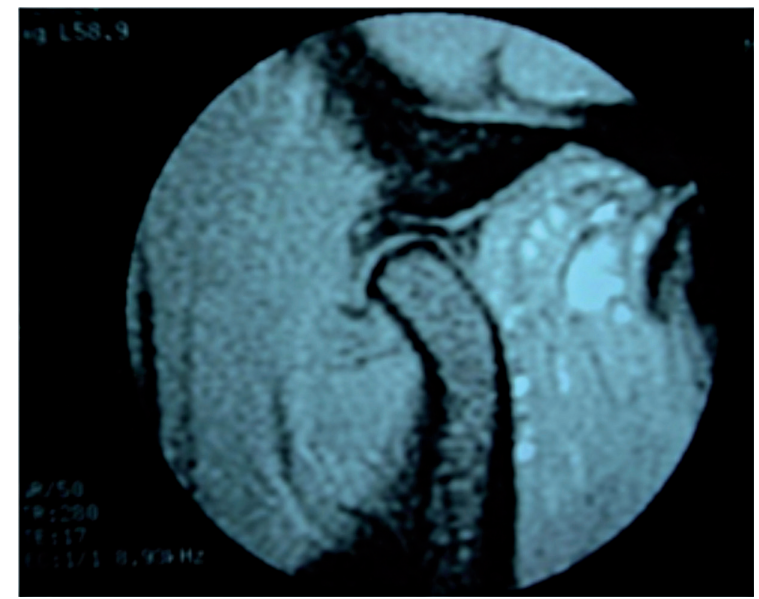

$\triangle$ Fig. 21 :

IRM bouche ouverte : le processus condylaire est à l'aplomb du tubercule articulaire du temporal. En ouverture buccale maximale, les processus condylaires viennent à l'aplomb du tubercule articulaire du temporal et déterminent louverture buccale physiologique propre à chaque patient. L'insertion du faisceau inférieur sur le processus condylaire est également visible (iconographie Jacques Dichamp).

et les faisceaux superficiels du M). Cette instabilité mécanique crée un terrain favorable dans la genèse des dysfonctions de l'ATM [7] mais son imputabilité n'est pas systématique en clinique car beaucoup de cas sont asymptomatiques.

Louverture buccale est jugée sur deux critères : I sa quantité ou amplitude d'ouverture mesurée appelée 


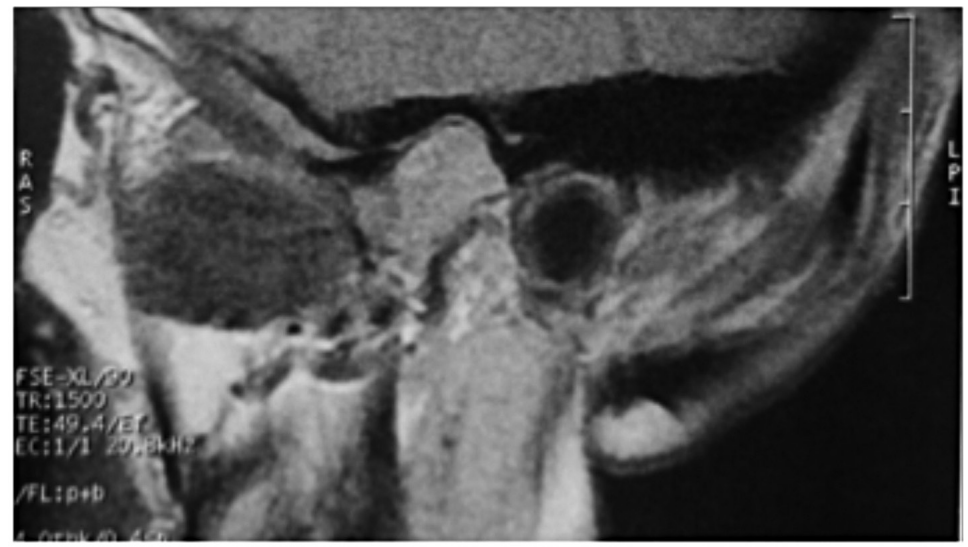

$\varangle$ Fig. 22 :

IRM dans le plan sagittal visualisant particulièrement la forme et l'orientation des deux faisceaux du PL : le faisceau supérieur disco-sphénoïdal est peu puissant par rapport au gros contingent de fibres musculaires rattaché au faisceau condylo-ptérygoïdien abaisseur de la mandibule. Cas clinique du service de stomatologie de l'hôpital européen Georges Pompidou.

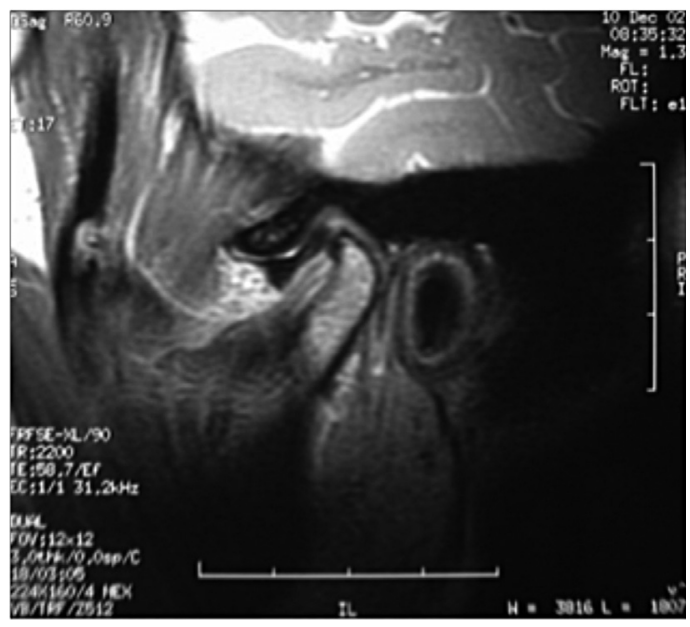

$\triangle$ Fig. 23 :

Cas clinique d'une dysfonction de l'ATM présentant un disque en avant du processus condylaire en bouche fermée entraînant une rétroposition du processus condylaire et une compression de la zone bilaminaire (iconographie Jacques Dichamp).

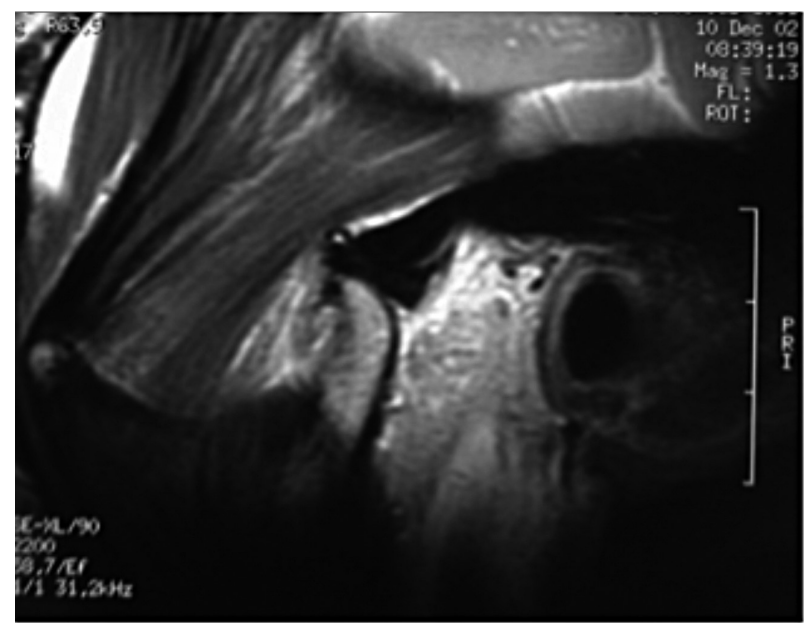

$\triangle$ Fig. 24 :

Le même cas montrant le caractère réductible de la luxation discale antérieure, le processus condylaire parvenant à l'aplomb du tubercule articulaire du temporal en bouche ouverte, sans altération de la cinétique. Noter la proximité des fibres postérieures du temporal avec le bord antérieur du disque articulaire (iconographie Jacques Dichamp). Certains cas sont réductibles en rotation interne : le processus condylaire contourne l'obstacle discal en diduction (action possible des muscles diducteurs controlatéraux), en général toujours au même endroit, pour pouvoir poursuivre l'ouverture buccale et lever le blocage. Ce dernier va généralement évoluer vers un blocage permanent en luxation discale irréductible [2].

distance interincisive maxillo-mandibulaire d'environ $45 \mathrm{~mm}$, donnée propre à chaque individu et importante dans le suivi au cas par cas des dysfonctions; I sa qualité rectiligne ou pathologique : latéro-déviée, sinusoïdale fixe qui traduit l'existence d'un obstacle sur le trajet du processus condylaire (disque en butée puis réduction toujours au même endroit de la pente condylienne par exemple) ou sinusoïdale labile qui traduit une incoordination musculaire (spasmes selon [4]).

\section{Anatomie fonctionnelle et imagerie}

La difficulté de visualisation de l'aspect dynamique des articulations temporo-mandibulaires constitue le problème majeur pour la compréhension de leur anatomie complexe (Fig. 18 à 24) [3]. En effet, structuralement parlant, l'ATM est composée de tissus durs et de tissus mous qui sont difficiles à explorer à l'aide d'une seule technique d'imagerie. Classiquement, l'imagerie tomodensitométrique par rayons $\mathrm{X}$ est particulièrement bien adaptée à la visualisation des tissus durs et l'imagerie par résonance magnétique est, quant à elle, particulièrement bien adaptée à la visualisation des tissus mous. On peut réunir les deux fichiers numériques créés par ces deux examens sur un même individu en établissant des repères de positionnement. Toutefois, la ciné-IRM n'est qu'une animation qui ne traduit malheureusement en aucun cas la cinétique en temps réel du processus condylaire mandibulaire, entraînant la genèse de modèles biomécaniques nécessairement incomplets. L'utilisation d'une cale pour maintenir 
louverture buccale lors de l'examen en bouche ouverte est habituelle à cause des temps de pose trop longs qui fatiguent le patient.

Les coupes sagittales de l'ATM sont les plus contributives et les plus usitées en imagerie pour apprécier la position du processus condylaire mandibulaire et de son disque par rapport à l'os temporal. Cette observation est nécessaire à la compréhension en anatomie descriptive et fonctionnelle, lors des phases délévation et d'abaissement, ainsi qu'en pathologie. Toutefois, l'ATM admet des mouvements dans toutes les directions de l'espace car elle travaille aussi en rotation, translation et diduction. Les coupes coronales et axiales sont donc également nécessaires à létude de son anatomie en imagerie 3D comme on peut les obtenir avec le CT SCAN ou l'IRM qui constituent les examens d'imagerie les plus prescrits en clinique à l'heure actuelle. La gravité ressentie à l'examen tomodensitométrique devant limportance des atteintes anatomiques n'est pas corrélée à l'intensité des phénomènes algiques décrits lors de l’anamnèse.

\section{CONCLUSION}

On ne note pas nécessairement de parallélisme anatomo-clinique du fait de l'adaptation fonctionnelle et de la tolérance, surtout algique, propre à chaque individu. Les douleurs spastiques peuvent ne pas avoir de correspondance anatomique objectivable par les examens d'imagerie actuels et ces derniers peuvent $a$ contrario révéler des anomalies ou des destructions importantes de l'articulation à caractère asymptomatique. L'innervation motrice et sensitive de l'ATM est assurée par le nerf mandibulaire, douleurs et spasmes musculaires s'exacerbant mutuellement lors des SADAM.

Les rapports otologiques se limitent anatomiquement à une innervation partagée entre le $\mathrm{PM}$, le tenseur du tympan et le tenseur du voile ainsi quà la présence d'une scissure pétro-tympano-squameuse en rapport direct avec l'articulation. L'imputabilité des symptômes ORL (acouphènes et problèmes tubaires) à un syndrome dysfonctionnel ne peut être retenue de façon systématique.

Lanatomie statique de l'ATM est bien décrite dans la littérature mais l’anatomie cinétique de cette articulation n'a pas encore livré tous ses secrets. Elle reste moins bien explorée en l'absence d'examens d'imagerie adaptés à un suivi des mouvements mandibulaires en temps réel. Une certaine variabilité individuelle dans l'anatomie descriptive de cette région échappe à l'archétype monolithique, "gravé dans le marbre ". Dans une sphère biomédicale gouvernée par la biologie moléculaire, la génétique et la biochimie, l’anatomie, cette "vieille dame", reste une valeur princeps pour diagnostiquer et traiter nos patients atteints de dysfonctions de l'appareil manducateur. Une amélioration de la connaissance et de la compréhension de l'anatomie fonctionnelle et dynamique des ATMs permettra dans le futur de mieux prendre en charge ces patients.

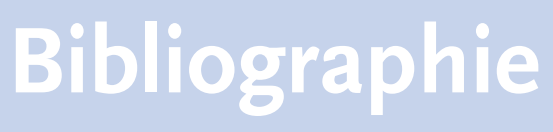

[1] Aoun M. Élaboration et validation d'un modèle de l'articulation temporo-mandibulaire par éléments finis. Thèse de doctorat en mécanique et ingéniérie.

Université Bordeaux I, 2010.

[2] Bonnefoy C. Dysfonctions de l'articulation temporo-mandibulaire. Compte-rendu de la conférence du jeudi 22 octobre 2009 présidée par le Dr Luc Chikhani. Actual Odonto-Stomatol 2010;250:189-202.

[3] Cavezian R, Pasquet G, Bel G, Baller G. Imagerie dento-maxillaire. Paris : Masson Elsevier, 2006.

[4] Chikhani L, Dichamp J. Bruxisme, Syndrome AlgoDysfonctionnel des Articulations Temporo-Mandibulaires et toxine botulique. Annales de réadaptation et de médecine physique 2003;46:333-337.
[5] Dargaud J, et al. Rapports de dissection sur la connaissance de l'articulation temporomandibulaire. EMC Masson-Elsevier, 2007 [23-446-C-15].

[6] Delaire J. Bases anatomiques et physio-pathologiques du traitement chirurgical des ankyloses temporo-mandibulaires. Rev Stomatol Chir Maxillofac Masson, 1983;1:19-26.

[7] Gambade S, Marteau J-M. Caix P. Rôle du muscle ptérygoïdien médial dans les dysfonctions cranio-mandibulaires. Med Buccale Chir Buccale 2006;12:73-82.

[8] Gaudy J.-F. et al. Anatomie clinique, $2^{\mathrm{e}}$ édition. Éditions CdP Wolters Kluwers France, 2007.

[9] Goudot P, Hérisson C. Pathologie de l'articulation-temporo-mandibulaire. Paris : Masson Elsevier, 2003.

[10] Gray H. Gray's Anatomy. New-York Barnes and Nobles $15^{e}$ édition, 2010.
[11] Lézy J-P, Princ G. Pathologie maxillofaciale et stomatologie. Paris : Masson Elsevier, $3^{e}$ édition, 2004.

[12] Norton N S. Précis d'anatomie clinique de la tête et du cou. Paris : Masson Elsevier, 2009.

[13] Maillot C, Kahn J-L. Tête et cou anatomie topographique. Paris : Springer France, 2004.

[14] Reychler H, Marashi R, Bialas C. Les douleurs de l'articulation temporo-mandibulaire, Louvain Med 119 : S490-S496, 2000.

[15] Rouvière H, Delmas A. Anatomie humaine, Tome 1 Tête et cou ( $15^{\mathrm{e}}$ édition). $654 p$. Masson-Elsevier. 2002.

[16] Rowicki T, Zakrzewska J. A study of the discomalleolar ligament in the adult human, Folia Morphol (Warsz), 2006;65(2):121-5.

[17] Vacher C. Anatomie clinique de l'articulation temporo-mandibulaire. Actual Odonto-Stomatol 2009;246:129-133. 